\title{
Burst-properties as a function of mass accretion rate in GX $3+1$
}

\author{
P. R. den Hartog ${ }^{1,2}$, J. J. M in 't Zand ${ }^{1,2}$, E. Kuulkers ${ }^{1,2, \star}$, R. Cornelisse ${ }^{1,2}$, J. Heise ${ }^{2,1}$, A. Bazzano ${ }^{3}$, M. Cocchi ${ }^{3}$, \\ L. Natalucci ${ }^{3}$, and P. Ubertini ${ }^{3}$ \\ 1 Astronomical Institute, Utrecht University, PO Box 80000, 3508 TA Utrecht, The Netherlands \\ 2 SRON National Institute for Space Research, Sorbonnelaan 2, 3584 CA Utrecht, The Netherlands \\ 3 Istituto Astrofisica Spaziale e Fisica Cosmica, CNR, via Fosso del Cavaliere 100, 00133 Roma, Italy
}

Received 16 October 2002 / Accepted 9 January 2003

\begin{abstract}
GX 3+1 is a low-mass X-ray binary that is persistently bright since its discovery in 1964. It was found to be an X-ray burster twenty years ago proving that the compact object in this system is a neutron star. The burst rate is so low that only 18 bursts were reported prior to 1996. The Wide Field Cameras on BeppoSAX have, through a dedicated monitoring program on the Galactic center region, increased the number of X-ray bursts from GX 3+1 by 61 . Since GX $3+1$ exhibits a slow (order of years) modulation in the persistent flux of about $50 \%$, these observations opens up the unique possibility to study burst properties as a function of mass accretion rate for very low burst rates. This is the first time that bursts are detected from GX $3+1$ in the high state. From the analysis we learn that all bursts are short with e-folding decay times smaller than $10 \mathrm{~s}$. Therefore, all bursts are due to unstable helium burning. Furthermore, the burst rate drops sixfold in a fairly narrow range of $2-20 \mathrm{keV}$ flux; we discuss possible origins for this.
\end{abstract}

Key words. accretion, accretion discs - binaries: close - stars: individual: GX 3+1 - stars: neutron - X-rays: bursts

\section{Introduction}

GX $3+1$ is one of the first discovered cosmic X-ray sources. It was detected during an Aerobee-rocket flight on June 16, 1964 (Bowyer et al. 1965). Ever since, GX 3+1 is one of the brightest persistent sources with an average $2-10 \mathrm{keV}$ flux half that of the Crab. Its X-ray intensity shows a mild variability on an hourly time scale and possibly a sinusoidal-like variation on a time scale of years (Makishima et al. 1983).

Although an optical counterpart has not yet been identified (e.g., Naylor et al. 1991), GX 3+1 is presumably a low mass $\mathrm{X}$-ray binary (LMXB) where a neutron star (NS) is accompanied by a low-mass star of spectral type A or later. In such a system (for a review, see White et al. 1995) the companion overflows its Roche lobe and dumps matter onto the NS through an accretion disc. The accumulation of matter on the NS surface leads to pressures and temperatures that are high enough to initiate nuclear fusion processes of the accreted hydrogen and helium to higher-Z nuclei (Hansen \& van Horn 1975). The circumstances of this process are such that the fusion may be unstable; only at accretion rates in excess of the Eddington limit is the burning thought to be completely stable (for solar abundances of the accreted matter; Fujimoto et al. 1981).

Send offprint requests to: P. R. den Hartog,

e-mail: P.R.den.Hartog@sron.nl

* Present address: ESA-ESTEC, SCI-SDG, Keplerlaan 1, 2201 AZ, Noordwijk, The Netherlands.
Unstable fusion occurs through thermonuclear flashes which may be observable in the form of X-ray bursts (Woosley \& Taam 1976; Maraschi \& Cavaliere 1977). Such so-called typeI X-ray bursts are characterized by a fast rise (one to ten seconds), exponential-like decay (e-folding decay times between a few seconds and tens of minutes), a black body spectrum of $k T \sim 1-3 \mathrm{keV}$, and cooling during the decay (for a review, see Lewin et al. 1993). For the flash to be observable, helium is essential as a fuel ingredient (Joss 1977). The amount of hydrogen involved in the burning layer determines the longevity of the explosion (e.g., Taam \& Picklum 1979). Currently, about half of all LMXBs have been seen to burst (e.g., in 't Zand 2001); GX 3+1 is one of them.

GX 3+1 was first seen to burst in 1983 (Makishima et al. 1983), about eight years after the discovery of the first X-ray burster (Grindlay \& Heise 1975), testifying to an infrequent bursting behavior. Up to now 20 bursts have been reported in the literature from GX 3+1: 15 with Hakucho (Makishima et al. 1983), 2 with Ginga (Asai et al. 1993), 1 with Granat (Pavlinsky et al. 1994) and 2 with the Rossi X-ray Timing Explorer (RXTE) (Kuulkers \& van der Klis 2000; Kuulkers 2002). Nineteen of these bursts are short with decay times less than $10 \mathrm{~s}$. The remaining burst, seen with the All Sky Monitor (ASM) on RXTE, is a superburst with a decay time of $\sim 1.6$ hours (Kuulkers 2002). One of the short bursts was seen to exhibit a quick (i.e., less than 2 s) radius expansion (factor of two) phase, indicating that the burst luminosity was 
near or at the Eddington luminosity, causing the NS atmosphere to expand due to radiation pressure. This implies a distance to the source of $4.5 \mathrm{kpc}$, for a hydrogen-rich NS atmosphere (Kuulkers \& van der Klis 2000).

In a simple picture a larger accretion rate would imply that ignition conditions are reached in quicker succession and burst rates should correspondingly be higher, under the assumption that the amount of fuel burned per burst is similar. Remarkably, the opposite is usually observed. In the majority of the roughly ten cases where this issue could be investigated (including GX 3+1) the burst frequency was found to be smaller at higher accretion rates, assuming that higher fluxes reflect higher accretion rates. Only in one case is there clear evidence for a positive correlation: GS 1826-24 (Cocchi et al. 2000). The anticorrelation between flux and burst frequency prompted the fuel "leakage" hypothesis: fuel is burnt in a non-observable way with a rate that increases with accretion rate (van Paradijs et al. 1988). Recently, Bildsten (2000) proposed as an alternative that the explosion may be occurring on only part of the NS surface, and that the expected proportionality for the burst frequency is with the accretion rate per unit area instead of the global accretion rate (see also Marshall 1982).

We here discuss observations of GX $3+1$ that were obtained with the Wide Field Cameras on BeppoSAX between 1996 and 2002. This data set is unique because of its sheer volume, giving rise to the detection of 61 bursts, which allows for a more detailed study of the infrequent bursting behavior of GX 3+1 than was hitherto possible. The merits of such a study are increased by the slow but large change in brightness over the course of these observations, enabling an analysis of the dependency of burst properties, such as recurrence times, on flux and possibly accretion rate.

\section{Observations}

\subsection{BeppoSAX observations}

The Italian-Dutch X-ray satellite BeppoSAX (Boella et al. 1997), launched on April 30, 1996, and switched off exactly six years later, carried two Wide Field Cameras (WFCs; Jager et al. 1997). They were active between 2 and $28 \mathrm{keV}$ and had field of views (FOVs) of $40^{\circ} \times 40^{\circ}$ with an angular resolution of $5^{\prime}$, and a source location accuracy of $0.7^{\prime}$ (99\% confidence). One of the core programs of BeppoSAX was a Galactic center monitoring program with the WFCs. The observations were made during two visibility windows per year, from mid February to mid April and from mid August to mid October (see Table 1 and Fig. 1). Since GX 3+1 is located close to the Galactic center, it was usually near the center of the FOV, so that the sensitivity was optimum. The complete dataset consists of 12 Galactic center campaigns, including serendipitous secondary observations near the Galactic center, with a total net exposure time of 6.2 Ms. In this dataset 61 bursts were identified with GX $3+1$. The bursts were found through analyzing the full bandpass (2$28 \mathrm{keV}$ ) light curves of GX $3+1$ at a time resolution of $5 \mathrm{~s}$. The peak flux detection threshold for bursts that have an e-folding decay time of at least $3 \mathrm{~s}$ is then expected to be $1 \mathrm{cts} \mathrm{cm}^{-2} \mathrm{~s}^{-1}$, or roughly half the flux of the Crab.

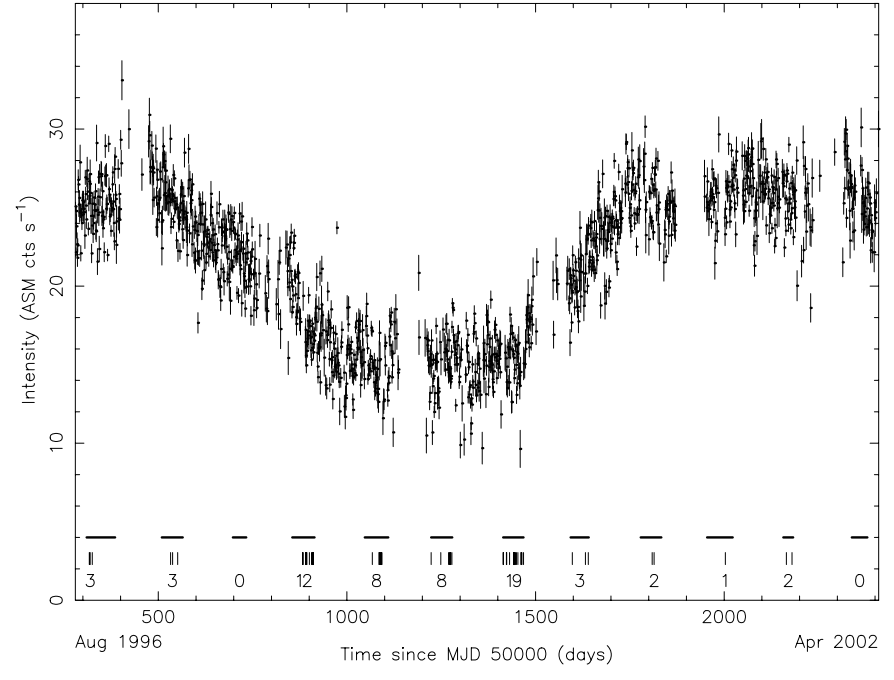

Fig. 1. ASM 1.5-12 keV light curve (one-day averages) from August 1996 to April 2002. For clarity, data points with error bars larger than $1.25 \mathrm{cts} \mathrm{s}^{-1}$ were not plotted (i.e., $16 \%$ of all points). Below the light curve the time spans of the twelve BeppoSAX Galactic center campaigns are shown with horizontal bars. Below the time spans the burst times are indicated with vertical lines, and we indicate the number of bursts per campaign.

\subsection{ASM observations}

The All Sky Monitor on board RXTE (Levine et al. 1996) monitored GX $3+1$ over the six years covering the WFC era (and beyond). Figure 1 shows the 1.5 to $12 \mathrm{keV}$ light curve. Plotted are one-day averages of the $90 \mathrm{~s}$ dwells taken by the ASM. It shows that the intensity of GX $3+1$ slowly changed by a factor of two. At first the source was in a "high state" with a flux of about 0.4 times that of the $\mathrm{Crab}\left(75 \mathrm{cts} \mathrm{s}^{-1}\right)$. Then the intensity dropped over the course of about 20 months until it reached a "low state" with a flux that is $50 \%$ lower. GX $3+1$ stayed in this low state for about 19 months after which the intensity slowly increased until it reached the high state after 10 months again. The reason for using the ASM data set is that it provides a more uniform coverage than the WFC data set. Thus, one can get a better idea how the source behaved between bursts when the recurrence time is longer than the typical WFC observation.

\section{Burst analysis}

A time-resolved spectral analysis was performed of all 61 bursts. The time resolution is limited by the statistical quality of the data, which is determined by the burst flux, duration and the off-axis angle. We required a resolution so that the 1 sigma errors in the spectral parameters remained within $20 \%$.

The 2-28 keV light curves, at a resolution of one second, served as a guide for setting up the time intervals for which burst spectra were extracted. The first interval was set to start when the photon flux showed a significant trend towards a clear peak. In this regard it is useful to note that all 61 bursts showed onsets that lasted less than three seconds. The end of the burst was defined as the instance when an exponential fit function to the decay dropped to the average persistent level within one 
Table 1. Summary of the twelve Galactic center observation campaigns of the WFCs. For every campaign it shows the campaign number, year, begin and end time $\left(t_{\text {begin }}\right.$ and $\left.t_{\text {end }}\right)$, the number of observations, the net exposure time $\left(t_{\exp }\right)$, the number of bursts seen from GX $3+1$ during that campaign, the derived burst rate and the average persistent ASM intensity $\left(\overline{F_{\text {pers }}}\right)$.

\begin{tabular}{cclllcccc}
\hline \hline Camp. & year & $t_{\text {begin }}$ & $t_{\text {end }}$ & \# obs. & $t_{\text {exp }}(\mathrm{ks})$ & \# bursts & burst rate $\left(\mathrm{Ms}^{-1}\right)$ & $\overline{F_{\text {pers }}}\left(\mathrm{cts} \mathrm{s}^{-1}\right)$ \\
\hline 1 & 1996 & Aug. 15 & Oct. 29 & 67 & 1017 & 3 & $2.9_{-1.6}^{+2.9}$ & $25.13 \pm 0.06$ \\
2 & 1997 & Mar. 02 & Apr. 26 & 21 & 654 & 3 & $4.6_{-2.5}^{+4.4}$ & $25.23 \pm 0.07$ \\
3 & 1997 & Sep. 06 & Oct. 12 & 13 & 302 & 0 & $<3.8$ & $22.22 \pm 0.09$ \\
4 & 1998 & Feb. 11 & Apr. 11 & 17 & 551 & 12 & $21.8_{-6.2}^{+8.2}$ & $18.25 \pm 0.07$ \\
5 & 1998 & Aug. 22 & Oct. 23 & 10 & 410 & 8 & $19.5_{-6.7}^{+9.6}$ & $14.98 \pm 0.07$ \\
6 & 1999 & Feb. 14 & Apr. 11 & 14 & 470 & 8 & $17.2_{-5.8}^{+8.3}$ & $14.92 \pm 0.08$ \\
7 & 1999 & Aug. 24 & Oct. 17 & 24 & 801 & 19 & $23.7_{-5.3}^{+6.7}$ & $15.50 \pm 0.08$ \\
8 & 2000 & Feb. 18 & Apr. 07 & 21 & 633 & 3 & $4.7_{-2.5}^{+4.6}$ & $20.47 \pm 0.09$ \\
9 & 2000 & Aug. 22 & Oct. 16 & 29 & 767 & 2 & $2.6_{-2.5}^{+3.4}$ & $25.82 \pm 0.09$ \\
10 & 2001 & Feb. 14 & Apr. 23 & 5 & 215 & 1 & $4.7_{-3.9}^{+10.6}$ & $25.64 \pm 0.07$ \\
11 & 2001 & Sep. 04 & Sep. 30 & 7 & 284 & 2 & $7.0_{-4.9}^{+8.9}$ & $25.57 \pm 0.08$ \\
12 & 2002 & Mar. 05 & Apr. 15 & 5 & 91 & 0 & $<12.5$ & $25.33 \pm 0.14$ \\
\hline
\end{tabular}

sigma. We also included two time intervals covering the persistent emission, namely a $10-15 \mathrm{~s}$ interval just before the burst onset and a 80-90 s interval from the end of the burst. In total three to five time bins were defined for each burst. For each time interval, dead-time corrected background-free spectra were extracted in the $2-28 \mathrm{keV}$ band. During the analysis we ignored channels 1, 2 and 31, and included a systematic error of $1.5 \%$ per channel, to accommodate calibration uncertainties.

For each burst, we assumed that the spectrum of the persistent emission was constant and modeled it with thermal bremsstrahlung. This is a simplification with respect to the more sophisticated models discussed in Sect. 5, but is allowed because 1) the statistical quality of the short (115 s) segments is insufficient to discriminate between models, and 2) we are not interested in the details of this spectrum at this point but merely in (implicitly) subtracting the persistent emission. The spectrum of the burst emission was modeled by a single-temperature black body, whereby the temperature and normalization were left free over all burst time intervals. On all spectral models, a fixed absorption was applied, following Morrison \& McCammon (1983), with $N_{\mathrm{H}}=1.59 \times 10^{22} \mathrm{~cm}^{-2}$ (Oosterbroek et al. 2001). This model fits the data satisfactorily $\left(\chi_{\mathrm{r}}^{2}\right.$ ranges up to 1.5 with at least 80 d.o.f. for at least 3 spectra). For each burst interval the fit provides an estimate for the radius $(R)$ of the emission area (i.e., of a sphere and assuming isotropical emission), the black body temperature $(k T)$ and the predicted unabsorbed bolometric flux $(F)$. An example of the results of the spectral analysis is shown in Fig. 2. Since the time bins are broad, the parameters are not very time sensitive. For instance, there is not a high enough temporal resolution to detect a radius expansion such as seen by Kuulkers \& van der Klis (2000), which lasted less than two seconds. Also the peak bolometric flux is lower than the true peak flux for the same reason. Therefore, in the remainder we will consider only the observed peak photon flux $\left(F_{\text {peak }}\right)$, since in this paper we only compare bursts seen by the WFC. The total fluence $\left(E_{\mathrm{b}}\right)$ is determined by integrating the mean observed fluxes over the time bins.
Figure 3 shows the burst parameters for all 61 bursts plotted versus time. To search for variability in each parameter, $\chi_{\mathrm{r}}^{2}$ was determined with respect to the weighted mean. This shows that the emission area is consistent with being constant $\left(\chi_{\mathrm{r}}^{2}=0.87\right)$. The weighted mean of its spherical radius is $4.46 \pm 0.08 \mathrm{~km}$ for a distance of $4.5 \mathrm{kpc}$, with a standard deviation of $0.44 \mathrm{~km}$. The non-variability suggests that the emitting radius area is the complete surface of the NS and not a variable fraction. However, the radius is small with respect to the expected value (e.g., Haensel 2001). Some of this may be explained by inverse Compton scattering in a hot electron cloud (see Ebisuzaki 1987). Also, we suspect that the distance estimate of $4.5 \mathrm{kpc}$ may be too low (see discussion). The other parameters do show variability, but only the variation in peak flux is (anti) correlated with the state of the persistent flux: when the source is in the low state it shows a larger dynamical range. The distribution of the peak fluxes in the low state is more or less Gaussian with a mean of $2.85 \mathrm{cts} \mathrm{cm}^{-2} \mathrm{~s}^{-1}$ and a variance of $0.35 \mathrm{cts} \mathrm{cm}^{-2} \mathrm{~s}^{-1}$. All the bursts in the high state have peak fluxes less than $3 \mathrm{cts} \mathrm{cm}^{-2} \mathrm{~s}^{-1}$. The low state distribution predicts a chance probability of $97.5 \%$ for seeing at least one burst with a peak flux higher than $3 \mathrm{cts} \mathrm{cm}^{-2} \mathrm{~s}^{-1}$ in the high state. Thus, the chance probability of not seeing a burst with a peak flux higher than $3 \mathrm{cts} \mathrm{cm}^{-2} \mathrm{~s}^{-1}$ in the high state is only $2.5 \%$. Therefore, bursts during the low state convincingly reach higher (i.e., up to two times) peak fluxes than during the high state. We can not say anything conclusive about the decay times $(\tau)$ other than that for all bursts the decay times are short (i.e., less than $8 \mathrm{~s}$ ) with a weighted mean of $3.63 \pm 0.10 \mathrm{~s}$. This is similar to the bursts from GX $3+1$ seen with other instruments except for the superburst. As mentioned in the introduction, the longevity of a burst is indicative of the amount of hydrogen mixture in the helium fuel for the flash. The above decay times suggest that we are dealing with pure helium burning. In order to double check this, we averaged all bursts separately for the low and the high state, see Fig. 4. Thus, the dynamic range of the flux measurements is increased by at least a factor of 4 . This figure shows that 1) there is no trace of 


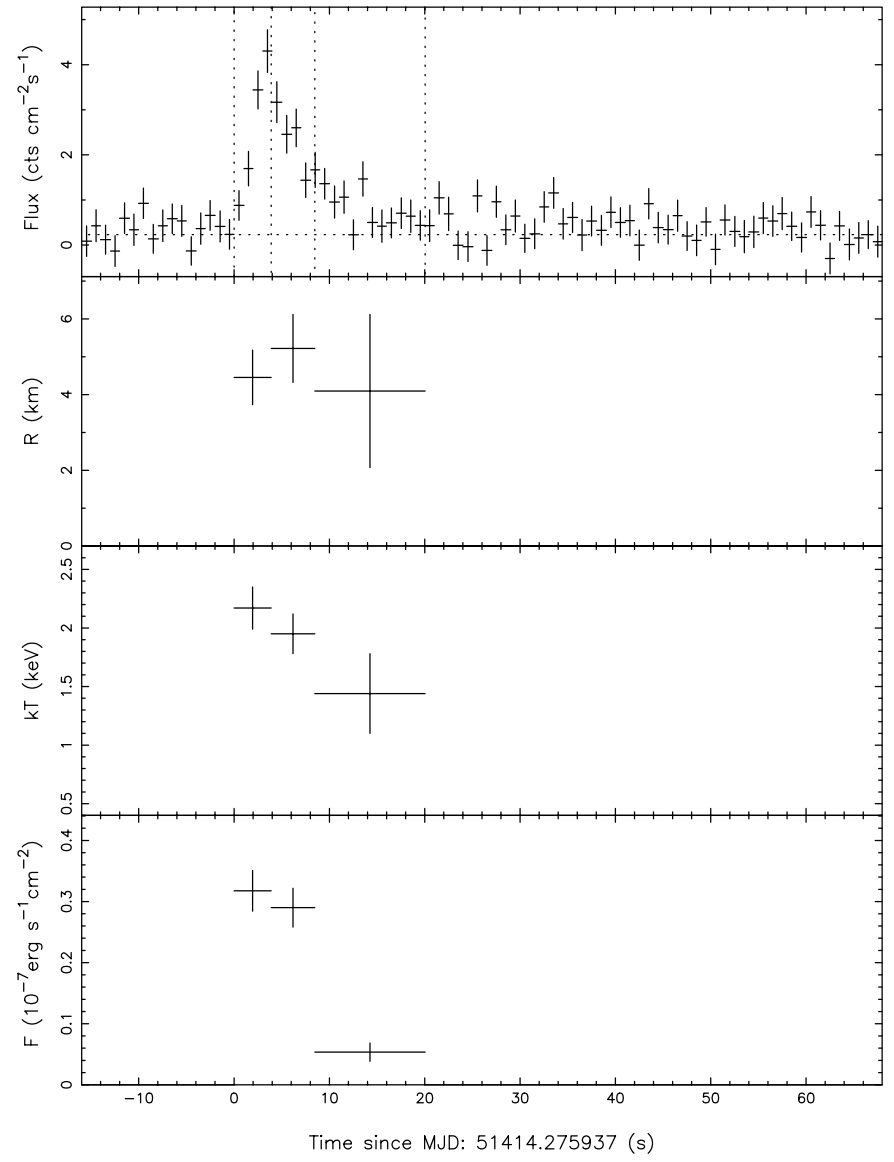

Fig. 2. Example of the results of the spectral analysis of a burst. In the top panel the $2-28 \mathrm{keV}$ light curve of the burst is shown. The horizontal dotted line is the determined persistent flux. The vertical dotted lines show the bin boundaries. The second panel shows the radii of the emitting region on the NS (assuming isotropic emission and a distance of $4.5 \mathrm{kpc}$ ). The radii of the three bins are constant over time. The third panel shows the black body temperature of the NS. In the bottom panel the average bolometric black body flux is shown.

prolonged burst emission and 2) the profiles are identical within the statistics provided by the data. For comparison, we added in Fig. 4 the average WFC-measured profile of all long bursts from GS 1826-24. This source is a well-documented exhibitor of mixed hydrogen/helium flashes (e.g., Bildsten 2000). These bursts are clearly a factor of roughly six longer than those of GX $3+1$. We conclude that all bursts from GX $3+1$ are short bursts which are thought to be the product of a thermonuclear runaway process that is fueled by pure helium.

\section{Burst rate}

We have investigated the burst rate as a function of the average persistent intensity $\left(\overline{F_{\text {pers }}}\right)$. To assess the exposure times during which the sensitivity was sufficient to detect bursts with peak fluxes in excess of $1 \mathrm{cts} \mathrm{s}^{-1} \mathrm{~cm}^{-2}$ (or $0.5 \mathrm{Crab}$ ) we excluded all the observations whenever the source was located within 30 pixels from the edge of the FOV. This amounts up to $6 \%$ of the total exposure. For the remaining data we derived the net exposure times, taking into account Earth occultations and passages over the South Atlantic Geomagnetic Anomaly

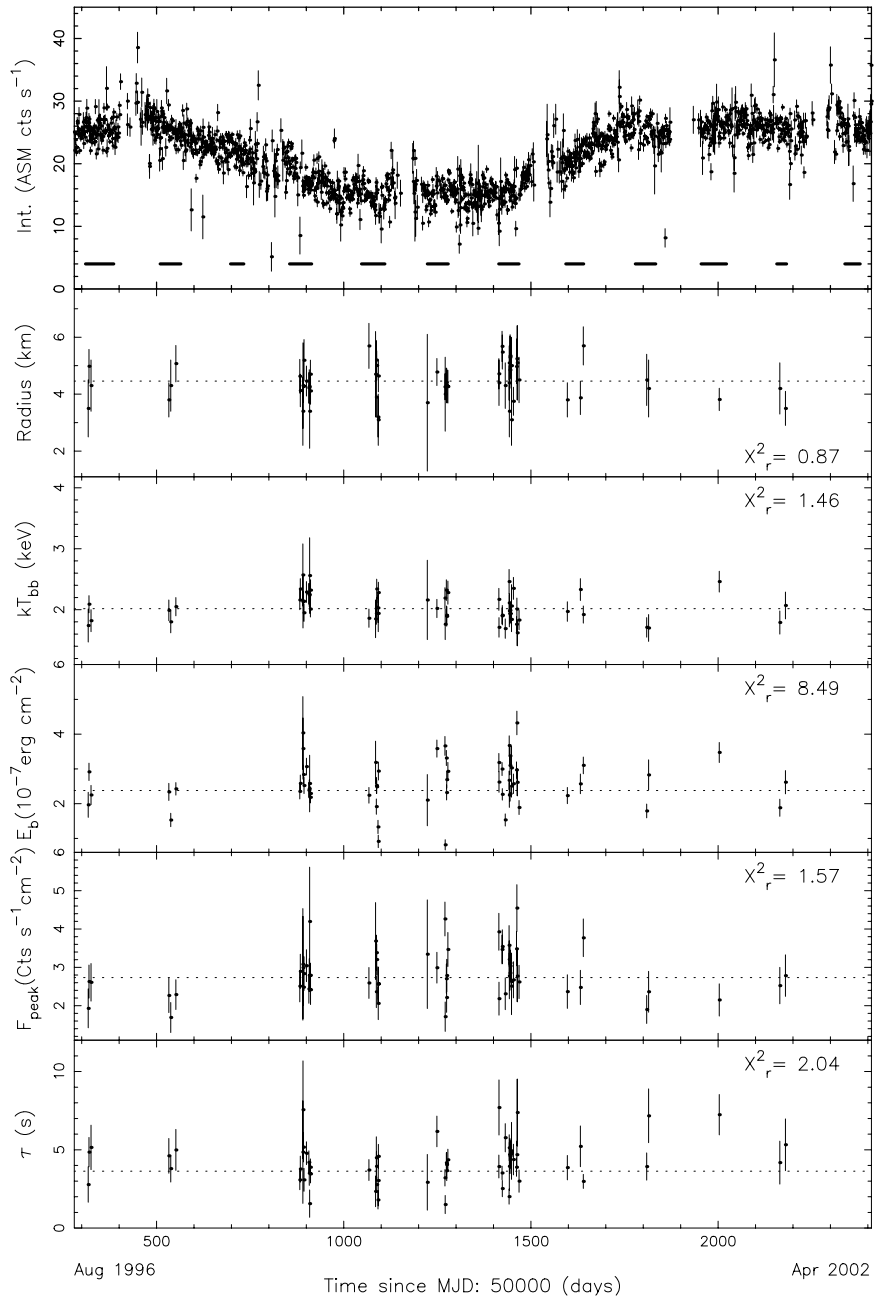

Fig. 3. In the top panel the ASM 1-12 keV light curve is shown together with the WFC coverage as horizontal bars. In the lower panels the burst properties versus time. From 2nd to lowest panel: radius of the emission area (assuming $4.5 \mathrm{kpc}$ distance), black body temperature, burst fluence, peak flux and decay time. The dotted lines are the weighted means of the properties. The radii per burst are the weighted means of all time bins, wile the temperature is derived from the first time bin since the burst onset.

(see $t_{\text {exp }}$ in Table 1). The burst rate was calculated by dividing the number of bursts by the net exposure time for each campaign. The $68 \%$ confidence margins were determined by assuming Poisson statistics. This results in asymmetric errors. As no bursts were seen during the third and the twelfth campaign only upper limits to the burst rates could be derived. They are 3.8 and $12.5 \mathrm{Ms}^{-1}$ (68\% confidence), respectively. For the complete list we refer to Table 1 . Since the persistent emission and the burst rate of the different campaigns are sometimes similar, we averaged them over several campaigns. Campaigns 5, 6 and 7 were averaged for the low state and campaigns 1, 2, 9, 10, 11 and 12 (a null observation) for the high state. Campaigns 3,4 and 8 are the campaigns in which the overall intensity decreased or increased, of which campaign 3 is a null observation. For every campaign we calculated the average persistent intensity by averaging the ASM measurements over the complete time span of the campaign. Thus, biases due to the variability 


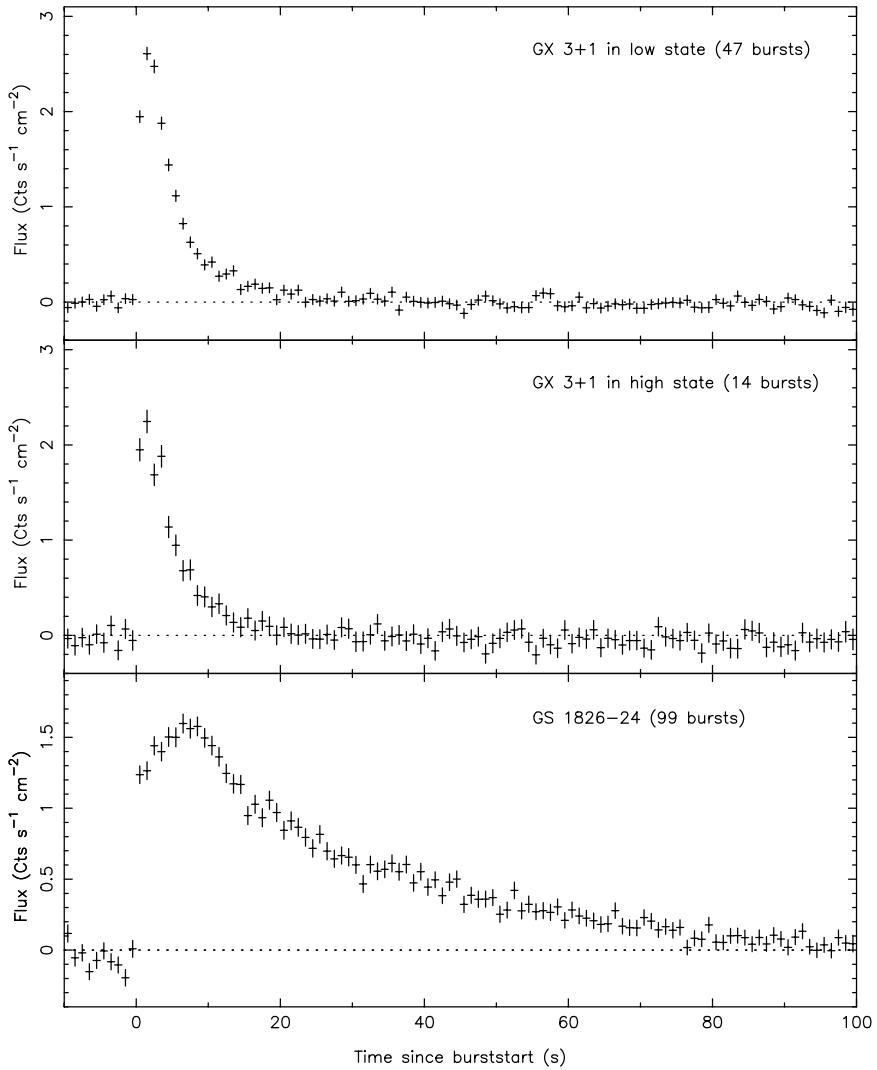

Fig. 4. Comparison of average $2-28 \mathrm{keV}$ burst profiles. From top to bottom, the average profiles of GX $3+1$ in the low state, GX $3+1$ in the high state and GS1826-24 are given. The average decay times are $4.23 \pm 0.13\left(\chi_{\mathrm{r}}^{2}=1.15\right), 4.97 \pm 0.28\left(\chi_{\mathrm{r}}^{2}=0.61\right)$ and $29.21 \pm 0.97$ $\left(\chi_{\mathrm{r}}^{2}=1.13\right)$ seconds, respectively.

on time scales shorter than the burst recurrence time were suppressed. In Fig. 5 the burst rate is plotted versus the persistent emission. At first sight, one can discriminate two levels of burst rate, with a quick transition from a high burst rate to a low burst rate at an intensity between $18.3 \mathrm{cts} \mathrm{s}^{-1}$ and $20.5 \mathrm{cts} \mathrm{s}^{-1}$. To test models for the behavior of the burst rate as a function of intensity one may not use the standard $\chi^{2}$-minimization, because the statistical fluctuations are not expected to be distributed as a Gaussian. Instead we chose to use the statistic

$S=\sum_{i=1}^{N} \frac{\left(d_{i}-m_{i}\right)^{2}}{m_{i}}$

where $d_{i}$ is the $i$ th measurement, $m_{i}$ the predicted measurement and $N$ the number of measurements $(N=5)$. $S$ was minimized through a grid search. Chance probabilities were evaluated through Monte Carlo simulations, by counting the number of times that simulated $S$-values were larger than measured $S$ values.

To find the most probable behavior three models were investigated: a constant, a linear and a step function. For the constant function no satisfactorily fit could be found, with a chance probability of $9 \times 10^{-6}$. For the linear model a better fit was found. For a function of the form $a+b x$ the values $a=46.46 \mathrm{Ms}^{-1}$ and $b=-1.69 \mathrm{Ms}^{-1} / \mathrm{cts} \mathrm{s}^{-1}$ were found. The

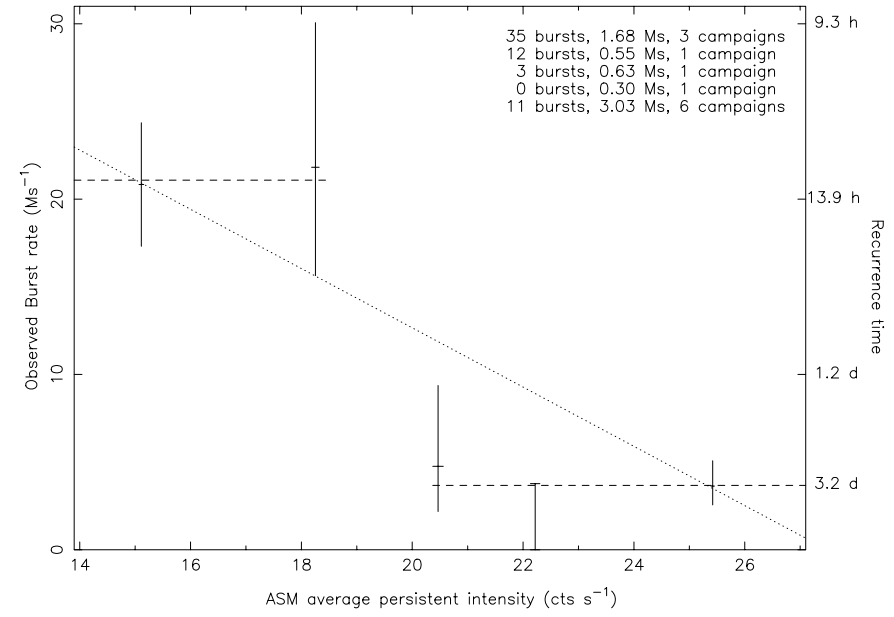

Fig. 5. Burst rate plotted versus the $1.5-12 \mathrm{keV}$ ASM intensity. The change in burst rate is a factor of six. The scale on the right hand side is the equivalent recurrence time for the specific burst rate ( $\mathrm{d}$ for days and $\mathrm{h}$ for hours). The dashed lines are the best fit of the high- and low burst rate level. The dotted line is the best straight line fit. In the upper right corner relevant information is given, namely the number of bursts, the net observation time and the number of campaigns for each data point from left to right.

chance probability is $21.6 \%$. The best fit is for the step function. The two levels that were found are $21.1 \pm 3.0 \mathrm{Ms}^{-1}$ and $3.67 \pm 1.0 \mathrm{Ms}^{-1}$. The chance probability is $95.3 \%$. Figure 5 shows the two fits. If the true relationship is a linear function, the chance that in a measurement like ours the minimum $S$ is smaller for a fit with a step function than for one with a linear function is $7.2 \pm 0.2 \%$, as shown by Monte Carlo simulations. This is the probability that we are dealing with a linear function rather than a step function.

In order to obtain an idea how the transition depends on the definition of the averaging of the persistent flux, we plot in Fig. 6 the burst rate as a function of the WFC average persistent flux. It shows that the transition between the high and low burst rate is less narrow. Also the distribution of the fluxes during the high state is broader. This is the reason why we have not plotted averages over campaigns like in Fig. 5. The broader transition is also apparent if we plot versus ASM flux if averaged over the times when the WFCs were on target rather than the complete time span of each campaign.

\section{Persistent emission and the mass accretion rate}

At time scales larger than one minute, GX 3+1 exhibits two kinds of variability (Asai et al. 1993). One has a time scale of order minutes to hours and the modulation depth in the 1$20 \mathrm{keV}$ intensity is typically $20 \%$. The intensity either correlates with the spectral hardness (when the source is said to be in the "banana" state) or not ("island" state). The 7-18/1-7 keV hardness ratio is observed to change by about $50 \%$. The other kind has a time scale of order years and is what causes the source to exhibit the so-called "low" and "high" states (Fig. 1). In the following we will concentrate on the latter (slow) variability because the minimum wait time between bursts is longer than the time scale of the faster variability; also the faster 


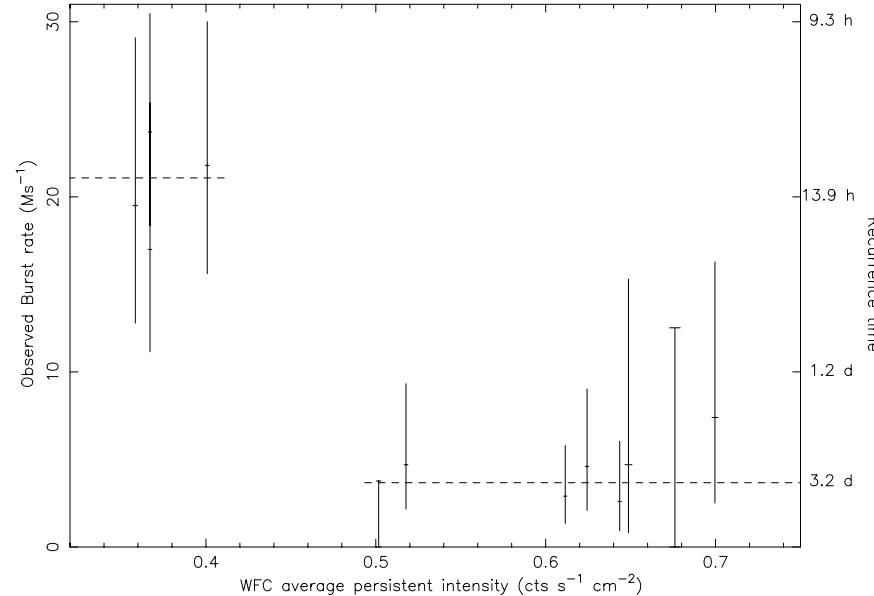

Fig. 6. Burst rate plotted versus the $2-28 \mathrm{keV}$ WFC flux. In essence is this the same plot as Fig. 5, but now the persistent flux is averaged over smaller time intervals. Every campaign has been plotted, because the spread in persistent intensity is larger. One sees that the transition between the high and low burst rate may be broader than in Fig. 5 (i.e., $20 \%$ in stead of $10 \%$ ).

variability is presumed to be unrelated to changes in the mass accretion rate (Asai et al. 1993). The slow variability has been shown nicely by 1987-1991 measurements with the all sky monitor on Ginga (Asai et al. 1993). The trends in those observations are quite similar to the ASM measurements. The same change in intensity is observed (a factor of 2); such a variation has been observed in earlier times as well (Makishima et al. 1983). The Ginga, ASM and earlier data sets even suggest that the flux oscillates semi-sinusoidally with a period of 6 to 7 years. Interestingly, the Ginga measurements with its Large Area Detector show a constant 1-20 keV spectral shape over the low and high states (Asai et al. 1993).

The stability of the X-ray spectrum over the low and high states is more or less confirmed by the WFC measurements in the 2-28 keV band in the sense that spectral changes are minimal, see Table 2 and Fig. 7. We made a selection of one long observation (i.e., longer than one day, except for the last campaign which did not include such a long observation) per campaign and investigated the full-resolution spectra. A general fit with an absorbed power law plus disk black body, fixing $N_{\mathrm{H}}$ again at $1.59 \times 10^{22} \mathrm{~cm}^{-2}$ (Oosterbroek et al. 2001) and coupling the disk black-body temperature and power law index over all 12 spectra, yields $\chi^{2} / v=1.31(v=310$; assuming a systematic uncertainty of $1.5 \%$ and ignoring the insufficiently calibrated spectral channels 1, 2 and 31 of each spectrum). If the temperature of the disk black body is allowed to vary over the 12 spectra, the fit improves to $\chi^{2} / v=1.027$ $(v=299)$. Uncoupling the power law index instead of the temperature yields a slightly worse fit with $\chi^{2} / v=1.06(v=299)$. Applying the same model as Oosterbroek et al. (2001) on the 0.5-200 keV NFI spectrum, leaving free only the fluxes of the 3 components, the optical depth and the black body temperature, but keeping them coupled over the 12 spectra except for the disk black body normalization, results in a reasonable though formally not acceptable fit: $\chi^{2} / v=1.354(v=320)$. When the disk black body temperature is allowed to vary, the fit improves to $\chi^{2} / v=0.945(v=309)$. Irrespective of the two models, the black body temperature changes only modestly by about $15 \%$ over the 12 spectra.

We conclude that the one-day averaged spectral shape shows only a very modest change over a time scale of years. This change is completely attributable to the disk black body component. This black body component is likely due to emission from the inner edge of the accretion disk and is probably driven by changes in the mass accretion rate (e.g., Barret 2001).

A parameter that is more meaningful than the $2-12 \mathrm{keV}$ photon count rate as measured with the ASM is the unabsorbed bolometric flux $F_{\text {bol }}$ because that should approximate the mass accretion rate more accurately. If the spectral model is extrapolated to beyond the WFC bandpass, $F_{\text {bol }}$ is estimated to vary between $8 \times 10^{-9}$ and $14 \times 10^{-9} \mathrm{erg} \mathrm{cm}^{-2} \mathrm{~s}^{-1}$ (see Table 2). Such an extrapolation is justified because a study of the $0.5-200 \mathrm{keV}$ spectrum by Oosterbroek et al. (2001), taken on MJD 51420-21, showed that $99 \%$ of the $1-200 \mathrm{keV}$ flux is contained within 1-20 keV. For a distance of $4.5 \mathrm{kpc}$ the flux translates to a bolometric luminosity of $2-3 \times 10^{37} \mathrm{erg} \mathrm{s}^{-1}$ $\left(7-12 \times 10^{37} \mathrm{erg} \mathrm{s}^{-1}\right.$ if at a canonical distance of $8.5 \mathrm{kpc}$, equal to that of the galactic center). The smallest observed flux $\left(8 \times 10^{-9} \mathrm{erg} \mathrm{cm}^{-2} \mathrm{~s}^{-1}\right)$ in combination with the smallest possible distance $(4.5 \mathrm{kpc})$ and the largest possible NS radius $(10 \mathrm{~km})$, while assuming a NS mass of $1.4 M_{\odot}$ and a luminosity given by $G M \dot{M} / R$, yields the minimum mass accretion rate in GX $3+1: 1.7 \times 10^{-9} M_{\odot} \mathrm{yr}^{-1}$ (or $10 \%$ of Eddington for a hydrogen-dominated NS atmosphere). It is likely that part of the liberated gravitational energy is released in another form than radiation. This emphasizes that our lower limit for the accretion rate is a firm one. We note that the mass accretion per unit area $\dot{m}$ can be estimated independently from the distance by $\left.\left(F_{\mathrm{bol}} R / G M\right)\left(d / R_{\mathrm{app}}\right)^{2}\right)$ (Bildsten 2000). For the neutron star canonical values $\dot{m}$ is above $2 \times 10^{4} \mathrm{~g} \mathrm{~cm}^{-2} \mathrm{~s}^{-1}$.

\section{Discussion}

Our observations of the burst properties as a function of persistent flux can be summarized as follows. Within a narrow range of persistent flux of about $10 \%$ (i.e., 18.3 to $20.5 \mathrm{ASM} \mathrm{cts} \mathrm{s}^{-1}$ ), the average burst frequency drops by a factor of 6 from once per 13.2 hours to once per 3.2 days. Though the best fit is a step function, we can not rule out a gradual transition. This is the first time that GX $3+1$ is seen to be burst active in the high intensity state (cf., Makishima et al. 1983; Asai et al. 1993). From all other burst properties only the peak flux seems to be (anti) correlated with the persistent flux. It occasionally shows higher values, by a factor of up to 2 , during the low state. In terms of the decay time, there is no difference between the low and high state (i.e., they are short) and all bursts appear to be predominantly fueled by helium. As noted in the introduction, an anti correlation between persistent flux and burst frequency is not unexpected. However, the issues that set GX 3+1 apart are the quickness of the transition and the fact that on both sides of the transition only helium bursts are seen. There is perhaps one other source, that we know of, that may show a similar effect but the number of bursts seen in the high state is only two (4U 1636-53; Lewin et al. 1987). The origin of the anti 
Table 2. Results of best-fit spectral models for selection of parameters. The fits were applied to twelve WFC spectra of GX $3+1$, one per campaign.

\begin{tabular}{|c|c|c|c|c|c|c|c|}
\hline$(1)$ & $(2)$ & (3) & (4) & $(5)$ & $(6)$ & (7) & $(8)$ \\
\hline $50319.77-50320.86$ & 10.7 & 9.14 & $2.291 \pm 0.013$ & $21.0 \pm 0.6$ & $2.174 \pm 0.023$ & $21.0 \pm 1.0$ & 13.7 \\
\hline $50551.19-50552.08$ & 10.6 & 9.07 & $2.255 \pm 0.010$ & $23.1 \pm 0.6$ & $2.140 \pm 0.023$ & $22.1 \pm 1.1$ & 13.6 \\
\hline $50725.44-50726.54$ & 8.95 & 7.74 & $2.234 \pm 0.012$ & $20.0 \pm 0.5$ & $2.081 \pm 0.027$ & $19.9 \pm 1.2$ & 11.6 \\
\hline $50910.10-50911.25$ & 6.31 & 5.46 & $2.155 \pm 0.019$ & $15.2 \pm 0.6$ & $1.861 \pm 0.041$ & $18.2 \pm 1.8$ & 8.3 \\
\hline $51057.24-51058.38$ & 6.03 & 5.15 & $2.127 \pm 0.026$ & $14.2 \pm 0.8$ & $1.768 \pm 0.044$ & $20.3 \pm 2.2$ & 7.8 \\
\hline $51270.08-51271.21$ & 6.60 & 5.62 & $2.211 \pm 0.020$ & $13.9 \pm 0.6$ & $1.938 \pm 0.041$ & $16.2 \pm 1.5$ & 8.5 \\
\hline $51422.68-51423.81$ & 5.95 & 5.19 & $2.109 \pm 0.020$ & $15.9 \pm 0.8$ & $1.779 \pm 0.045$ & $20.1 \pm 2.2$ & 7.9 \\
\hline $51618.05-51619.21$ & 8.33 & 7.22 & $2.158 \pm 0.016$ & $20.4 \pm 0.7$ & $1.966 \pm 0.029$ & $22.6 \pm 1.5$ & 10.9 \\
\hline $51802.28-51803.42$ & 7.98 & 7.87 & $2.256 \pm 0.011$ & $17.2 \pm 0.4$ & $2.089 \pm 0.032$ & $16.4 \pm 1.1$ & 10.3 \\
\hline $51989.20-51989.88$ & 11.5 & 9.76 & $2.336 \pm 0.022$ & $21.2 \pm 1.1$ & $2.240 \pm 0.030$ & $20.3 \pm 1.2$ & 14.6 \\
\hline $52181.79-52182.83$ & 11.1 & 9.54 & $2.299 \pm 0.010$ & $22.8 \pm 0.5$ & $2.203 \pm 0.023$ & $21.0 \pm 1.0$ & 14.3 \\
\hline $52353.24-52353.71$ & 9.72 & 8.34 & $2.293 \pm 0.016$ & $20.1 \pm 0.8$ & $2.179 \pm 0.029$ & $18.3 \pm 1.1$ & 12.5 \\
\hline
\end{tabular}

(1) Observation time span (MJD); (2) unabsorbed 2-28 keV flux (in units of $10^{-9} \mathrm{erg} \mathrm{cm}^{-2} \mathrm{~s}^{-1}$ ); (3) unabsorbed 2-10 keV flux (in units of $\left.10^{-9} \mathrm{erg} \mathrm{cm}^{-2} \mathrm{~s}^{-1}\right)$; (4) diskbb $k T_{\text {in }}(\mathrm{keV})$; (5) diskbb $R_{\mathrm{in}}^{2} \cos (\theta)\left(\mathrm{km}^{2}\right)$; (6) and (7) as (4) and (5) except in combination with a Comptonized spectrum instead of a power law; (8) unabsorbed 0.01-100 keV flux predicted from the latter model (in units of $10^{-9} \mathrm{erg} \mathrm{cm}^{-2} \mathrm{~s}^{-1}$ ).

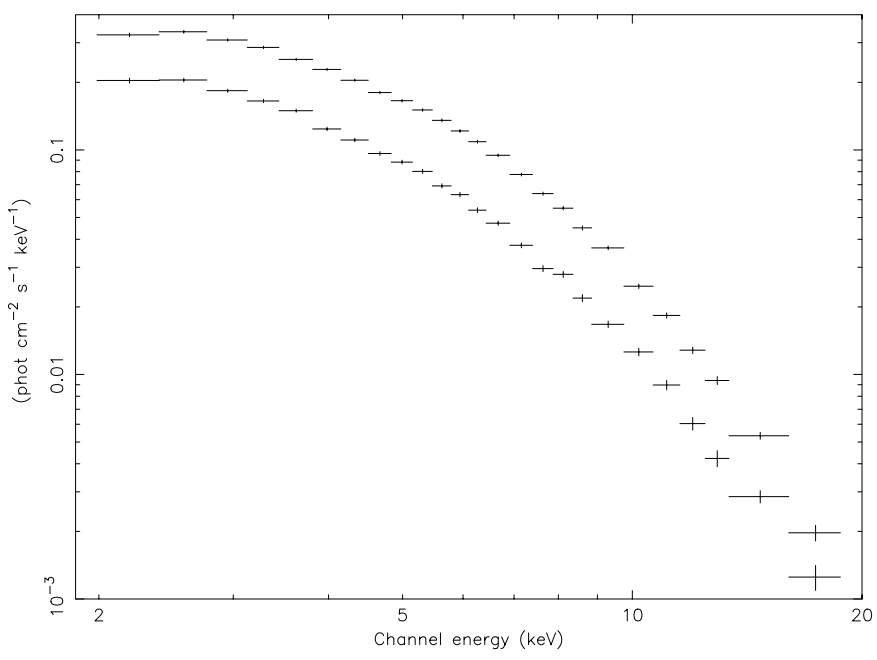

Fig. 7. Examples of incident photon spectra during low state and high state, as measured with the WFC on MJD 51057.24-51058.38 and MJD 52181.79-52182.83 respectively.

correlation is not well understood. The fact that in GX $3+1$ the anti correlation takes place over a rather small range of persistent flux may perhaps be an important clue.

The discrete-like nature of the transition in burst rate is reminiscent of the boundaries predicted by theory between regimes of unstable nuclear burning on accreting NSs (Fujimoto et al. 1981). For mass accretion rates $\dot{M}>2 \times 10^{-10} M_{\odot} \mathrm{yr}^{-1}$, the accreted hydrogen-rich material is expected to undergo stable hydrogen burning. At the bottom, a helium layer builds up in which the pressure increases because of the continuing accretion. At a certain moment the density becomes high enough to ignite the helium in an unstable burning process giving rise to a thermonuclear flash. If $\dot{M}<(4-11) \times 10^{-10} M_{\odot} \mathrm{yr}^{-1}$, the pressure is high enough for helium ignition only in the helium layer; however for higher $\dot{M}$ 's, the bottom of the hydrogen layer may also have sufficient pressure. As a result, the flash takes place in a mixture of helium and hydrogen and proton captures occur by the nuclear products of the helium burning which makes the flash extended because of the longer time scale of the resulting $\beta$ decays (Taam \& Picklum 1979). Below $2 \times 10^{-10} M_{\odot} \mathrm{yr}^{-1}$, the accreted hydrogen no longer undergoes stable burning, but unstable burning may occur at lower temperatures because of pressure build-up by the accumulating matter. The heating produced by this runaway process may ignite the helium accreted from the companion, provided the mass of the accumulated layer is large enough. In general, the amount of hydrogen participating in the flash is larger in this regime than in the regime $\dot{M}>2 \times 10^{-10} M_{\odot} \mathrm{yr}^{-1}$. Finally, there is a regime above roughly $10^{-8} M_{\odot} \mathrm{yr}^{-1}$ where even the helium is burned in a stable manner. This is near the Eddington limit and may not be observable.

The above regimes can experimentally be confirmed through measurements of burst durations. All observable bursts involve helium burning. For pure helium, the burst durations are predicted to be of order $10 \mathrm{~s}$, while the rise times are shorter than two seconds. Whenever hydrogen participates, the burst duration becomes longer. van Paradijs et al. (1988) confirmed the correspondence between theory and measurement, although for a limited data set. A particularly nice illustration of the regimes in a single source is $4 \mathrm{U}$ 1705-44 (Langmeier et al. 1987).

In GX $3+1$, the minimum $\dot{M}$ that is consistent with the persistent flux is $1.7 \times 10^{-9} M_{\odot} \mathrm{yr}^{-1}$. This is near or slightly above the upper boundary of the range where pure helium bursts are expected to occur. Therefore, the data appear discrepant with theory, even if abundances are sub-solar. Still, the factor-oftwo dynamic range of the persistent flux in GX 3+1 fits comfortably in the factor-of-five dynamic range predicted by theory for helium bursts. How do other bursters behave that have similar or higher persistent luminosities? Only a minority of bright LMXBs burst; we can think of the following four cases. Cyg X-2 is a Z-source with a luminosity close to Eddington. Thus far, 18 bursts were detected (Kahn \& Grindlay 1984; Kuulkers et al. 1995; Smale 1998; Titarchuk \& Shaposhnikov 2002). All are short, like in GX 3+1. The only other Z-source that exhibits bursts is GX 17+2 (e.g., Kuulkers et al. 2002). In that case seven out of ten bursts are clear examples involving 
hydrogen burning. Other sources are Cir X-1 (Tennant et al. 1986a,b) and GX 13+1 (Matsuba et al. 1995). While Cir X-1 shows somewhat longer bursts, the two bursts detected from GX $13+1$ are again short. We propose that the regime of mixed hydrogen/helium bursts at high accretion rates is seldom observed, that the regime with pure helium bursts may extend up to higher mass accretion rates, and that the transition of burst rate in GX $3+1$ is not due to change of regime.

The usual explanation for the anti-correlation is that a larger part of the helium is burned in a stable manner in the high state (Makishima et al. 1983; van Paradijs et al. 1988). Thus, less fuel is burnt in thermonuclear flashes. This is supported by the measurement of the burst parameter $\alpha$ (e.g., Lewin et al. 1993) which is the ratio of the fluence of the persistent emission from burst to burst and that of the burst. For the low state the average value is $1.7 \times 10^{3}$ and for the high state $2.1 \times 10^{4}$. Both these values are much higher than what is expected and is normal for most X-ray bursters (between 25 and 250). It is a strong indication that stable helium and/or hydrogen fusion is happening in both the low and the high state (van Paradijs et al. 1988). From the ratio in $\alpha$ the suggestion is that the energy production of the stable fusion which goes into radiation increases 12 -fold from the low to the high state. Stable helium burning is predicted to be possible only at near-Eddington accretion rates or above, while GX $3+1$ accretes at most at $30 \%$ of Eddington (see below). However, this difficulty could be circumvented by assuming that accretion takes place on a confined area of the NS (Fushiki \& Lamb 1987; Bildsten 2000). Anyway, if stable helium fusion is the process responsible for the anti-correlation, our detection of the rather discrete nature of this anti-correlation should provide an important test to theory.

There are the open issues of the higher peak fluxes during the low state, the comparatively small distance for a source that has such a small angular separation from the galactic center, and the doubtful small radii for the burst emission areas. We propose that they may all be resolved in the following manner. Ebisuzaki et al. (1983) determined that radiation from helium bursts may be intense enough to expel the hydrogen atmosphere, thereby leaving a hydrogen-poor photosphere. Such a photosphere has a higher Eddington limit so that luminosities may reach higher values by roughly a factor of two. This is more likely to happen if the hydrogen-dominated atmosphere is thinner. This is precisely what applies during the low state: the accretion rate is twice as small and the bursts recur six times quicker so that the accumulated mass from the companion between bursts is roughly twelve times smaller. Thus, this provides an explanation why peak fluxes are higher during the low state. The same explanation has recently been applied to the bimodal peak flux distribution of bursts from GX 354-0 by Galloway et al. (2002). Unfortunately, in our case we are not able to confirm the radius-expansion phase in the brighter bursts due to insufficient statistics.

However, the only radius-expansion burst from GX 3+1 detected by Kuulkers \& van der Klis (2000) has a peak flux of 2.3 Crab and happened during the low state. The peak flux compares well, within a few tens of percent, with the maximum peak flux that we find during the low state. The above reasoning implies that this burst reaches the Eddington limit for a helium-rich photosphere. Kuulkers \& van der Klis (2000) determined that in that case $(X=0)$ the distance would be $6.1 \pm 0.1 \mathrm{kpc}$. This, in comparison to the $4.5 \mathrm{kpc}$, is substantially closer toward the galactic center (at $8.5 \mathrm{kpc}$ ) and seems more plausible. Furthermore, the radius of the burst emission scales up accordingly to a more plausible value of $6.05 \pm 0.11 \mathrm{~km}$ (weighted mean). The luminosity of the persistent emission would increase accordingly. The maximum luminosity would be roughly $6 \times 10^{37} \mathrm{erg} \mathrm{s}^{-1}$, or $30 \%$ of Eddington. We note that $6.05 \mathrm{~km}$ is still smaller than the expected NS radius (Haensel 2001). This may be explained by inverse Compton scattering effects as discussed by Ebisuzaki (1987) which may introduce a factor of 2 decrease in the apparent radius for the black-body temperatures seen in GX $3+1$.

Acknowledgements. We would like to thank colleagues at the BeppoSAX Science Operations Center for their devoted support and the ASM/RXTE team for the repair of the light curve of GX $3+1$. $\mathrm{JZ}$ and EK acknowledge financial support from the Netherlands Organization for Scientific Research (NWO).

\section{References}

Asai, K., Dotani, T., Nagase, F., et al. 1993, PASJ, 45, 801

Barret, D. 2001, Adv. Space Res., 28, 307

Bildsten, L. 2000, in Cosmic Explosions: Tenth Astrophysics Conference., ed. S. Holt, \& W. Zhang (Melville: AIP), AIP Conf. Proc., 522, 359

Boella, G., Butler, R. C., Perola, G. C., et al. 1997, A\&AS, 122, 299

Bowyer, S., Byram, E. T., Chubb, T. A., \& Friedman, H. 1965, Science, 147, 394

Cocchi, M., Bazzano, A., Natalucci, L., et al. 2000, in The Fifth Compton Symposium, ed. M. L. McConnell, \& J. M. Ryan (Melville: AIP), AIP Conf. Proc., 510, 203

Ebisuzaki, T. 1987, PASJ, 39, 287

Ebisuzaki, T., Hanawa, T., \& Sugimoto, D. 1983, PASJ, 35, 17

Fujimoto, M. Y., Hanawa, T., \& Miyaji, S. 1981, ApJ, 247, 267

Fushiki, I., \& Lamb, D. Q. 1987, ApJ, 323, L55

Galloway, D. K., Psaltis, D., Chakrabarty, D., \& Muno, M. P. 2002, ApJ, submitted [astro-ph/0208464]

Grindlay, J., \& Heise, J. 1975, IAU Circ., 2879, 1

Haensel, P. 2001, A\&A, 380, 186

Hansen, C. J., \& van Horn, H. M. 1975, ApJ, 195, 735

in 't Zand, J. 2001, in ESA SP-459, Exploring the Gamma-Ray Universe, ed. A. Gimenez, V. Reglero, \& C. Winkler (Noordwijk: ESA Publications Division), 459

Jager, R., Mels, W. A., Brinkman, A. C., et al. 1997, A\&AS, 125, 557

Joss, P. C. 1977, Nature, 270, 310

Kahn, S. M., \& Grindlay, J. E. 1984, ApJ, 281, 826

Kuulkers, E. 2002, A\&A, 383, L5

Kuulkers, E., Homan, J., van der Klis, M., Lewin, W. H. G., \& Méndez, M. 2002, A\&A, 382, 947

Kuulkers, E., \& van der Klis, M. 2000, A\&A, 356, L45

Kuulkers, E., van der Klis, M., \& van Paradijs, J. 1995, ApJ, 450, 748

Langmeier, A., Sztajno, M., Hasinger, G., Truemper, J., \& Gottwald, M. 1987, ApJ, 323, 288

Levine, A. M., Bradt, H., Cui, W., et al. 1996, ApJ, 469, L33

Lewin, W. H. G., Penninx, W., van Paradijs, J., et al. 1987, ApJ, 319, 893

Lewin, W. H. G., van Paradijs, J., \& Taam, R. E. 1993, Space Sci. Rev., 62, 223 
Makishima, K., Mitsuda, K., Inoue, H., et al. 1983, ApJ, 267, 310

Maraschi, L., \& Cavaliere, A. 1977, Highlights of Astronomy, 4, 127 Marshall, H. L. 1982, ApJ, 260, 815

Matsuba, E., Dotani, T., Mitsuda, K., et al. 1995, PASJ, 47, 575

Morrison, R., \& McCammon, D. 1983, ApJ, 270, 119

Naylor, T., Charles, P. A., \& Longmore, A. J. 1991, MNRAS, 252, 203

Oosterbroek, T., Barret, D., Guainazzi, M., \& Ford, E. C. 2001, A\&A, 366,138

Pavlinsky, M. N., Grebenev, S. A., \& Sunyaev, R. A. 1994, ApJ, 425, 110

Smale, A. P. 1998, ApJ, 498, L141
Taam, R. E., \& Picklum, R. E. 1979, ApJ, 233, 327

Tennant, A. F., Fabian, A. C., \& Shafer, R. A. 1986a, MNRAS, 221, $27 \mathrm{P}$

Tennant, A. F., Fabian, A. C., \& Shafer, R. A. 1986b, MNRAS, 219, 871

Titarchuk, L., \& Shaposhnikov, N. 2002, ApJ, 570, L25

van Paradijs, J., Penninx, W., \& Lewin, W. H. G. 1988, MNRAS, 233, 437

White, N. E., Nagase, F., \& Parmar, A. N. 1995, in X-ray binaries, ed. W. H. G. Lewin, J. van Paradijs, \& E. P. J. Van den Heuvel (Cambridge, MA: Cambridge University Press), 1

Woosley, S. E., \& Taam, R. E. 1976, Nature, 263, 101 